U. S. Department of the Interior

Geological Survey

\title{
INTERPRETATION OF TIME-DOMAIN ELECTROMAGNETIC SOUNDINGS \\ IN THE CALICO HILLS AREA, \\ NEVADA TEST SITE, NYE COUNTY, NEVADA
}

by

James Kauahikaua

Open-File Report 81-988

1981

This report is preliminary and has not been reviewed for conformity with U. S. Geological Survey editorial standards, Any use of trade names is for descriptive purposes only and does not imply endorsement by the USGS. 


\title{
INTERPRETATION OF TIME-DOMAIN ELECTROMAGNETIC SOUNDINGS \\ IN THE CALICO HILLS AREA, \\ NEVADA TEST SITE, NYE COUNTY, NEVADA \\ by James Kauahikaua
}

\begin{abstract}
A controlled source, time-domain electromagnetic (TDEM) sounding survey was conducted in the Calico Hills area of the Nevada Test Site (NTS). The goal of this survey was the determination of the geoelectric structure as an aid in the evaluation of the site for possible future storage of spent nuclear fuel or high-level nuclear waste. The data were initially interpreted with a simple scheme that produces an apparent resistivity versus depth curve from the vertical magnetic field data. These curves can be qualitatively interpreted much like standard Schlumberger resistivity sounding curves. Final interpretation made use of a layered-earth Marquardt inversion computer program (Kauahikaua, 1980). The results combined with those from a set of Schlumberger soundings in the area show that there is a moderately resistive basement at a depth no greater than 800 meters. The basement resistivity is greater than $100 \mathrm{ohm}$-meters.
\end{abstract}

\section{Introduction}

Between June 2 and 8, 1978, nine TDEM soundings were completed in the Calico Hills area of the Nevada Test Site using a grounded-wire current source and a cryogenic magnetometer sensor. The source wire was 2,250 m long oriented along a direction $\mathrm{N} 78 \mathrm{~W}$, and continuously pulsed with a $4-$ to 6-amp current which changed polarity at $5 \mathrm{~s}$ intervals. The same source was used for all nine soundings. At each numbered location shown in Figure 1 , 
a three-component cryogenic SQUID magnetometer ( $\mathrm{X}$-axis parallel to the source wire) was set up to measure the magnetic field components generated by the switched current source. The magnetometer was partially buried and its top was covered with a plastic container to minimize wind noise. The vertical magnetic field $(\mathrm{Hz})$ was digitized and recorded on a Gould data logger at 200 - samples per second for a period of $4 \mathrm{~min}$ at each location. Horizontal fields perpendicular to the source wire (Hy) were similarly recorded at sounding locations $1,5,7,8$, and 9 . Natural magnetic noise was 1 ow during the survey period, allowing much of the recording to be done without electronic filtering. Field operations were directed by Dick Sneddon (USGS, Denver, Colorado).

Figure 1 also shows the locations of eight Schlumberger soundings that are interpreted in this study. The vertical electric sounding (VES) data were provided by Don Hoover, USGS (written communication, 1979).

\section{TDEM Data Reduction}

The data-logger tape cartridges were transcribed to 9-track tape so that data reduction could be done on the Honeywell Multics computer maintained by the USGS in Denver, Colorado. Each 4-min record should contain 40 to 50 step responses and have a total of about 50,000 data points. The easiest way to stack this data is to search it for the start of the response (look for large first differences), store the next $5 \mathrm{~s}$ of data, search for the start of the next response, etc., until the whole 50,000 data points have been searched. The search-and-store step will convert the data string of 50,000 points into a 50-by-1000 matrix of points - each of the 50 rows is an individual step response, and each of the 1,000 columns correspond to the responses at a particular time relative to the source switching time. 


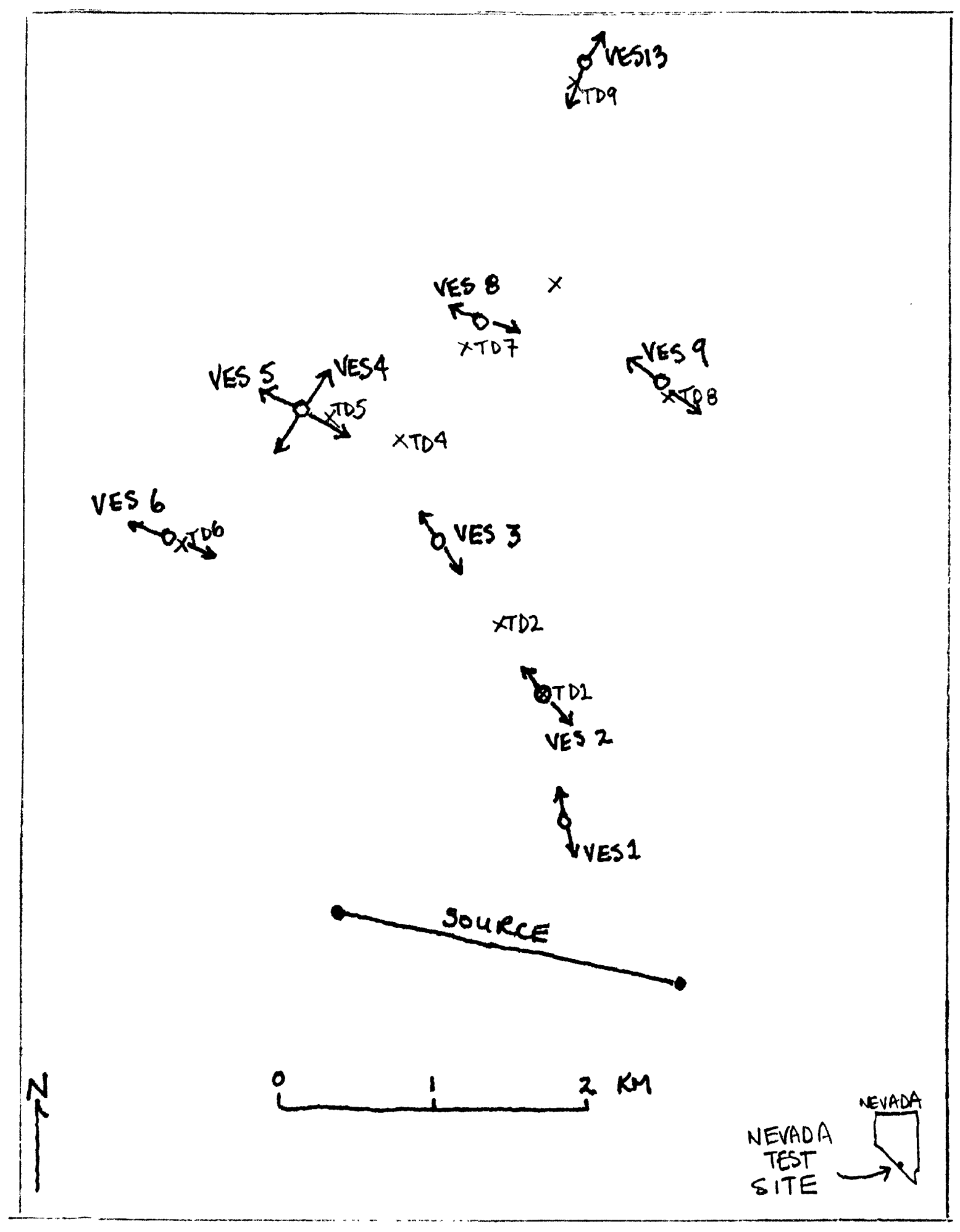

Figure 1: A topographic map of the Calico Hills area of the Nevada Test Site showing the locations of the grounded wire source and the nine cryogenic magnetometer receiver sites (prefixed by TD) used in this TDEM survey, Double-headed arrows are VES locations (prefixed by VES). 
The stacking proceeds by computing an average and a standard deviation for each of the 1,000 columns. If data from any of the step responses fall more than two standard deviations from the average, those data are rejected and the average and standard deviations are recomputed. The stacking step, as described above, produces an average response and a corresponding standard deviation for each of the 1,000 columns. Finally, the stacked response is smoothed with a time-varying filter which emphasizes low frequencies at late times and higher frequencies at early times. The final reduced response data set consists of 49 averaged magnetic field values (and the corresponding standard deviations) spaced at logarithmically equal intervals of time.

The response data were not corrected for the response of the system. This is usually a standard step in TDEM data reduction; however, the numerical means available for deconvolution of the data can be unstable, particularly with data taken over resistive terrain, such as the Nevada Test Site. The approach preferred in this work is to use the system response as part of the model to which we are trying to match the data. This is numerically more stable and does not significantly increase the computations.

\section{Data Interpretation Methods}

The first stage in interpretation is to familiarize oneself with the TDEM responses to simple models. Figures 2 and $3 b$ are two-layer step response models for a conductivity contrast (sig2/sig1) of 10 and $1 / 10$ and $\mathrm{d} / \mathrm{r}$ (ratio of first layer thickness to sourcersensor separation) values of $.05, .1, .25, .5$, and 1 . The modeled source is a horizontal electric dipole excited by a step function of current, and the received signal is not filtered. TDEM mode1 responses were computed with a program written by Kauahikaua and Anderson (1977). Figure 2a shows the horizontal magnetic 

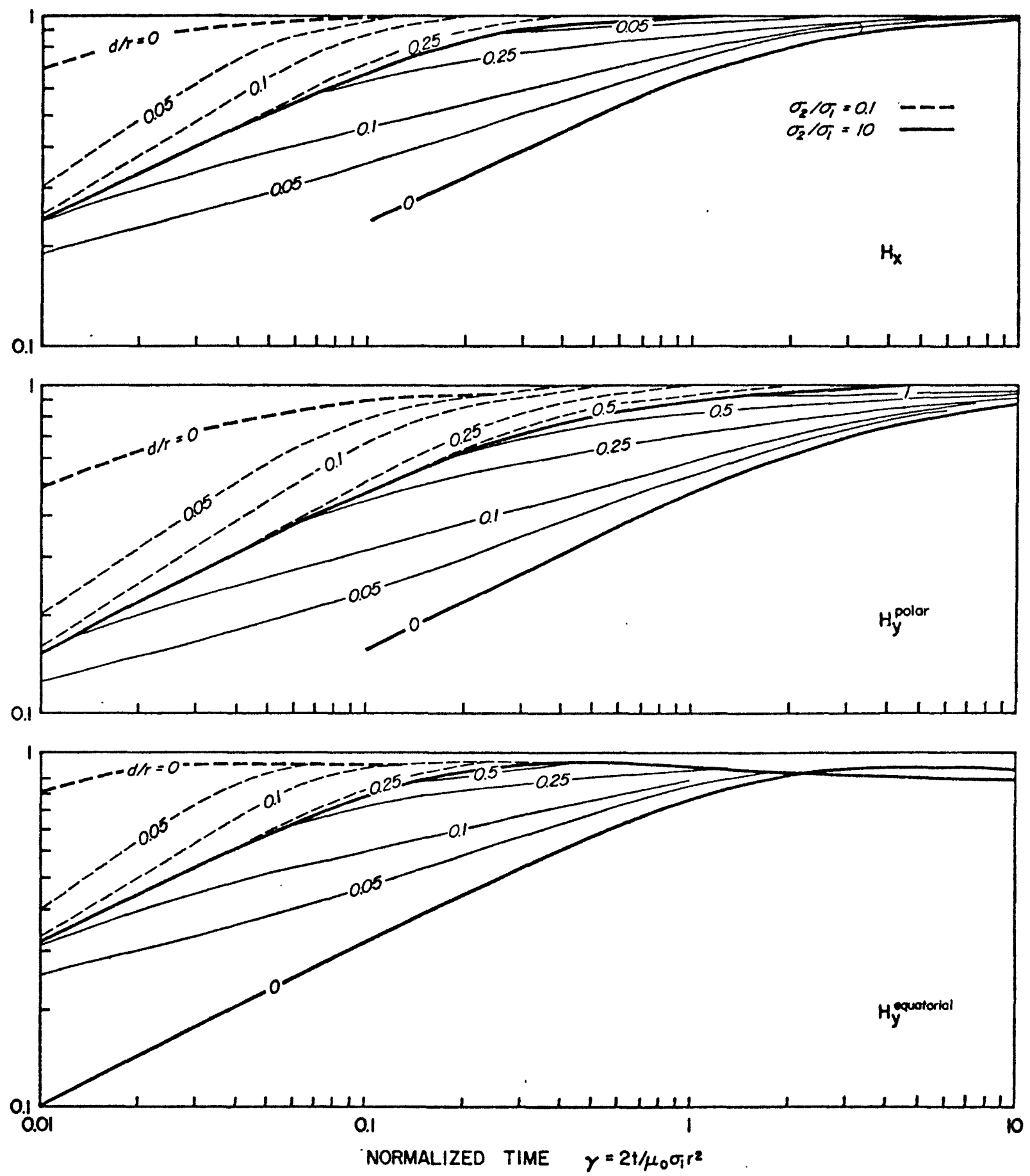

Figure 2. Plots of the theoretical transient horizontal magnetic field generated by a step current pulsed through a horizontal electric dipole. The fields are normalized by the appropriate free-space field values. Each figure shows model responses for two different conductivity contrasts of 10 and $1 / 10$ and values of $\mathrm{d} / \mathrm{r}$ (ratio of first layer thickness to source-sensor separation) of $0.0,0.05,0.1,0.25,0.5,1.0$, and infinity. a) magnetic field measured parallel to the dipole source, b) magnetic field measured perpendicular to the dipole source at a distance directly along the dipole direction, and c) magnetic field measured perpendicular to the dipole source at a distance measured perpendicular to the dipole direction. 

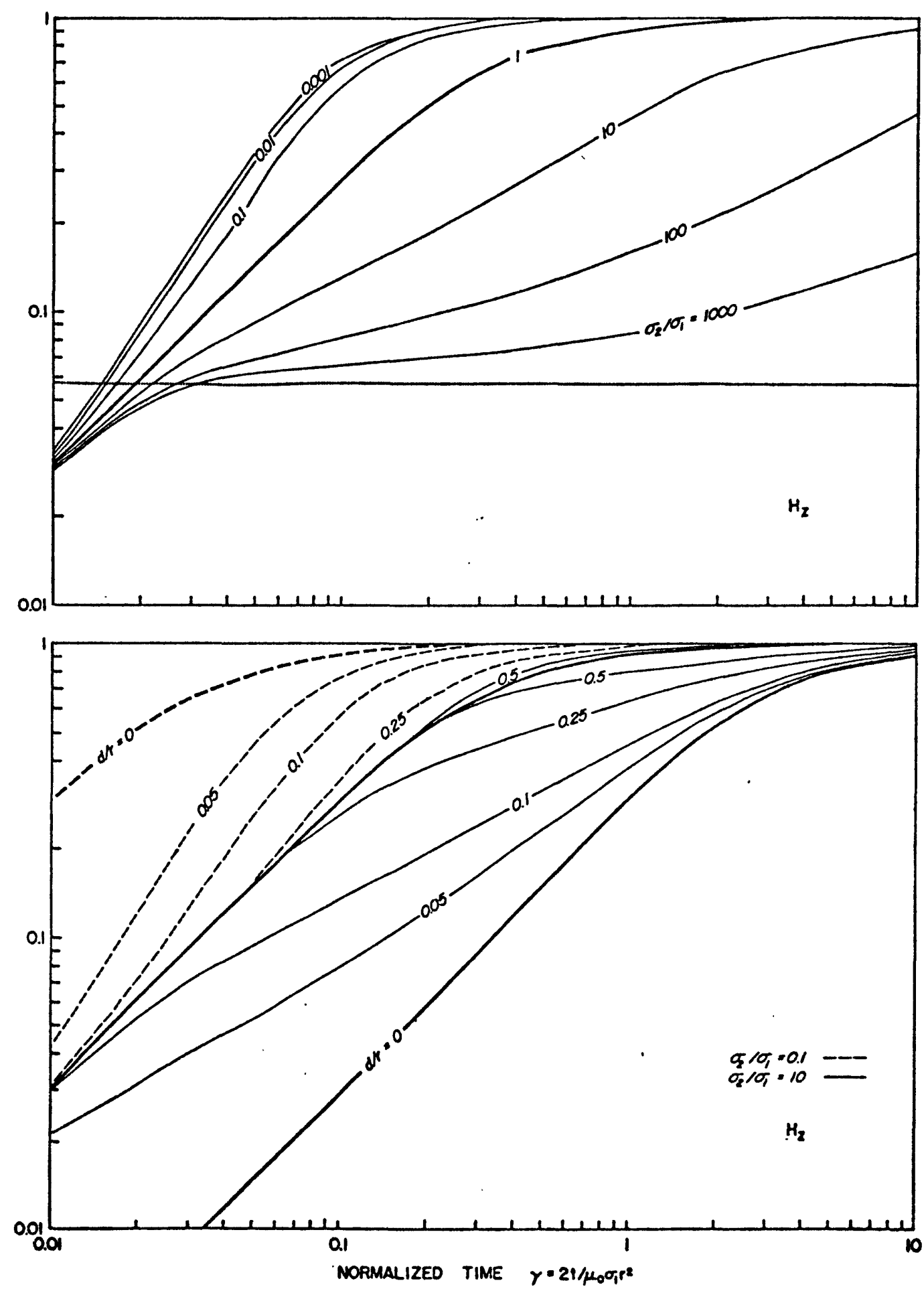

Figure 3. Plots of the theoretical transient vertical magnetic field generated by a step current pulsed through a horizontal electric dipole. The fields are normalized by the vertical free-space field value, a) first layer thickness is $1 / 10$ of the source-sensor separation and the conductivity contrast varies from $10 * *-3$ to $10 * * 3$. The dashed line is the asymptote for a conductivity contrast of infinity, and b) conductivity contrast of 10 and $1 / 10$ and a d/r ratio of $0.0,0.05,0.1,0.25,0.5,1.0$, and infinity. 
field in a direction parallel to the source dipole, and Figures $2 \mathrm{~b}$ and $2 \mathrm{c}$ show the directionally-dependent horizontal magnetic field perpendicular to the source dipole. Figure $3 b$ shows the vertical magnetic field. Note that the differences between the model responses are noticeably smaller for the horizontal fields than for the vertical field. This is because for early times, the horizontal fields build-up to the primary field value like $5 * * 0.5$ (1) whereas the vertical field builds up like $t$, where $t$ is time. A half-space conductivity change of 10 times would change the vertical field by a factor of 10 in early time, but would only change the horizontal fields by $10 * * 0.5=3$. Therefore, to a first-order approximation, the vertical magnetic field of a horizontal electric dipole is about 3 times more sensitive to changes in earth conductivity structure than are the horizontal magnetic fields. It would seem most profitable to concentrate on the vertical magnetic field data.

At this point, the best fitting layered earth model could be determined directly by inversion using program MQLVTHXYZ (Kauahikaua, 1980); however, this should be avoided if possible because typical computer runs are expensive. Quite a good estimate of the electrical structure can be obtained by converting the TDEM data into apparent resistivities as a function of time (Morrison and others, 1969). That is, at successive times in the TDEM response, one can calculate "what the resistivity of a homogeneous half space would have to be to yield the observed field amplitude at that time". This can be done graphically as will be demonstrated first with the halfspace response in Figure $3 b$, then with the actual data plots in Appendix $I$.

(1) exponentiation is denoted by ' $* *$ ' 
Computation of Apparent Resistivity from TDEM Data

First the data are normalized by the asymptotic late-time value. The date should then consist of values between zero and one. Next, the normalized time corresponding to each one of the normalized data points should be obtained using the half-space model curve in Figure 3B. Each of the normalized times can then be converted to an apparent resistivity using the corresponding real time and the source-sensor distance. As an example, let's use the data from a hypothetical sounding recorded at a distance of $1,000 \mathrm{~m}$ from a wire source. A normalized field value of 0.5 was measured at $70 \mathrm{~ms}$ after a break in the source current. Using Figure $3 \mathrm{a}$, we see that a field value of 0.5 corresponds to a normalized time of 0.18 . The equation defining normalized time in terms of real time is

$$
\text { normalized_time }=2 * r e a l \_t i m e * r h o /(m y u 0 * r * r)
$$

where $\mathrm{r}$ is source-sensor distance in $\mathrm{m}$, myu0 is $4 * \mathrm{pi} * 10 * *(-7)$, pi is 3.1415927, and tho is the half-space resistivity. Using the actual sourcesensor distance of $1,000 \mathrm{~m}$, the resistivity of the half space that would have produced a normalized vertical magnetic field value of 0.5 at $70 \mathrm{~ms}$ is $1.62 \mathrm{ohm}-\mathrm{m}$. This is the apparent resistivity at $70 \mathrm{~ms}$ for this point of the example sounding,

Taking this one step further and in the process approximately accounting for the system response, one can make a log-log graph paper with various half-space responses already plotted on it. The reduced data can be plotted on this customized graph paper and the apparent resistivity for each data point can be logarithmically interpolated using the two nearest half-space curves. For this study, the log-log graph paper has real time on its horizontal axis and normalized vertical magnetic field on the vertical axis. Half-space responses are 
plotted on it for various values of the ratio $r h o / R * * 2$ ( $R$ in kilometers). Because two different recording system configurations were used in the NTS study, two different types of customized graph paper were constructed. Some graphs were prepared using undistorted half-space responses and others were prepared using half-space responses distorted by a twin-T 60 Hertz notch filter. Apparent resistivities can even be estimated in the field in this way.

All nine vertical field TDEM sounding data sets are plotted in Appendix I on graph paper prepared in this manner. Each of the half-space responses have been labeled with the actual resistivity corresponding to the product of the rho/R**2 value of the half-space response and the square of that sounding's source-sensor distance, in kilometers. After logarithmically interpolating between half-space response curves for each data point, each apparent resistivitiy versus time function shows that the apparent resistivity increases with time. There is a slight decrease in resistivity at times greater than $50 \mathrm{msec}$ for soundings 1 and 2, and at times greater than $100 \mathrm{msec}$ for soundings 6 and 7. The exact depths to which these resistivities correspond can only be determined by modeling the TDEM responses themselves. The rule-of-thumb is that data at later times is information from greater depths. The NTS soundings are then depicting a structure with a conductive layer over a resistive one, with soundings $1,2,6$ and 7 suggesting another conductive layer at still greater depths.

Computation of Apparent Depth of Penetration

This difficulty with quantitative depth estimates led to the concept of apparent depth of penetration as a function of normalized magnetic field amplitude. The idea behind this concept can be described most simply using 
the two-layer model vertical magnetic field response curves in Figures $3 a$ and $3 \mathrm{~b}$. Figure $3 a$ shows responses to models with a fixed $d / \mathrm{r}$ ratio of $1 / 10$ and varying conductivity ratios, and Figure $3 \mathrm{~b}$ shows responses to models with fixed conductivity ratios of 10 and $1 / 10$ and varying $d / r$ ratios. These responses all show a conspicuous tendency to increase linearly with time from zero along the half-space response curve corresponding to the resistivity of the surface layer. The normalized field value at which these model curves depart from the half-space curve followed at early times can be seen to be characteristic of the particular $d / r$ ratio of the model, and therefore could be used to estimate maximum penetration depth at a particular time.

A mathematical definition of this maximum penetration depth can be obtained from the model response of a perfect resistor overlying a perfect conductor. The significance of this particular model is that it is the most resolvable of all earth models for electromagnetic systems. The normalized vertical magnetic-field response for such a model can be shown to be

$$
\mathrm{Hzn}=1-(4 *(\mathrm{~d} / \mathrm{r}) * 2+1) * *(-3 / 2) \text {. }
$$

The field does not vary with time because of the choice of perfect conductors and resistors in the model. To show how this relates to the model responses with finite resistivities, the value of this function for $d / r=0.1$ is plotted on Figure $3 \mathrm{a}$ as a horizontal dashed line, Obviously this is also the asymptotic (late-time) value of the induced vertical magnetic field for twolayer models with a finite-resistivity layer over a perfect conductor. For the case of $d / r=0.1$, less-than-perfectly conducting lower half spaces significantly distort the response curve from that of a half space with the first-layer resistivity only at times greater than the time at which that half-space response intersects the dashed line. This is at a normalized 
time of about 0.02 . In general, the above equation can be solved for the $\mathrm{d} / \mathrm{r}$ ratio, and each value in a set of TDEM response data could be converted to a $d / r$ value as well as an apparent resistivity. The physical meaning of these $d$ values is the maximum penetration depth at a given time for a given shallow resistivity structure. The $d / r$ ratio will be called the normalized apparent depth of penetration. The normalized apparent resistivity versus normalized apparent depth curves corresponding to the set of two-1ayer model responses in Figure $3 \mathrm{~b}$ are presented in Figure 4 as dashed lines. For comparison, Schlumberger model curves for the same two-layer models are plotted as solid lines in Figure 4. The Schlumberger electrode spacings $(\mathrm{AB} / 2)$ have been normalized by $r$, the TDEM source-sensor distance, for ease of comparison. The great similarity between the two sets of curves suggests that the apparent depth conversion for TDEM data could be as diagnostic as the Schlumberger curves.

The shape of the TDEM apparent resistivity versus depth curves seems to depend only upon the parameters of the earth model and not on $r$, the source-sensor separation. An album of model curves for TDEM sounding could be constructed much like they have been for Schlumberger sounding. They would require a greatly reduced number of curves compared to the normal set of EM model curves which are commonly related to $r$. The same album of curves would also be applicable to frequency-domain sounding using the field amplitude data. This is because there is nothing implicitly time-or frequency-domain oriented about the approach except in the nature of apparent resistivity calculation (substitute reciprocal of $2^{*}$ pi*frequency for time). The same approach could also be used in other systems (frequency- or timedomain) which employ electromagnetic fields. In this way, TDEM data can be compensated for the system response and reduced to a pseudo-Schlumberger form in the field. 


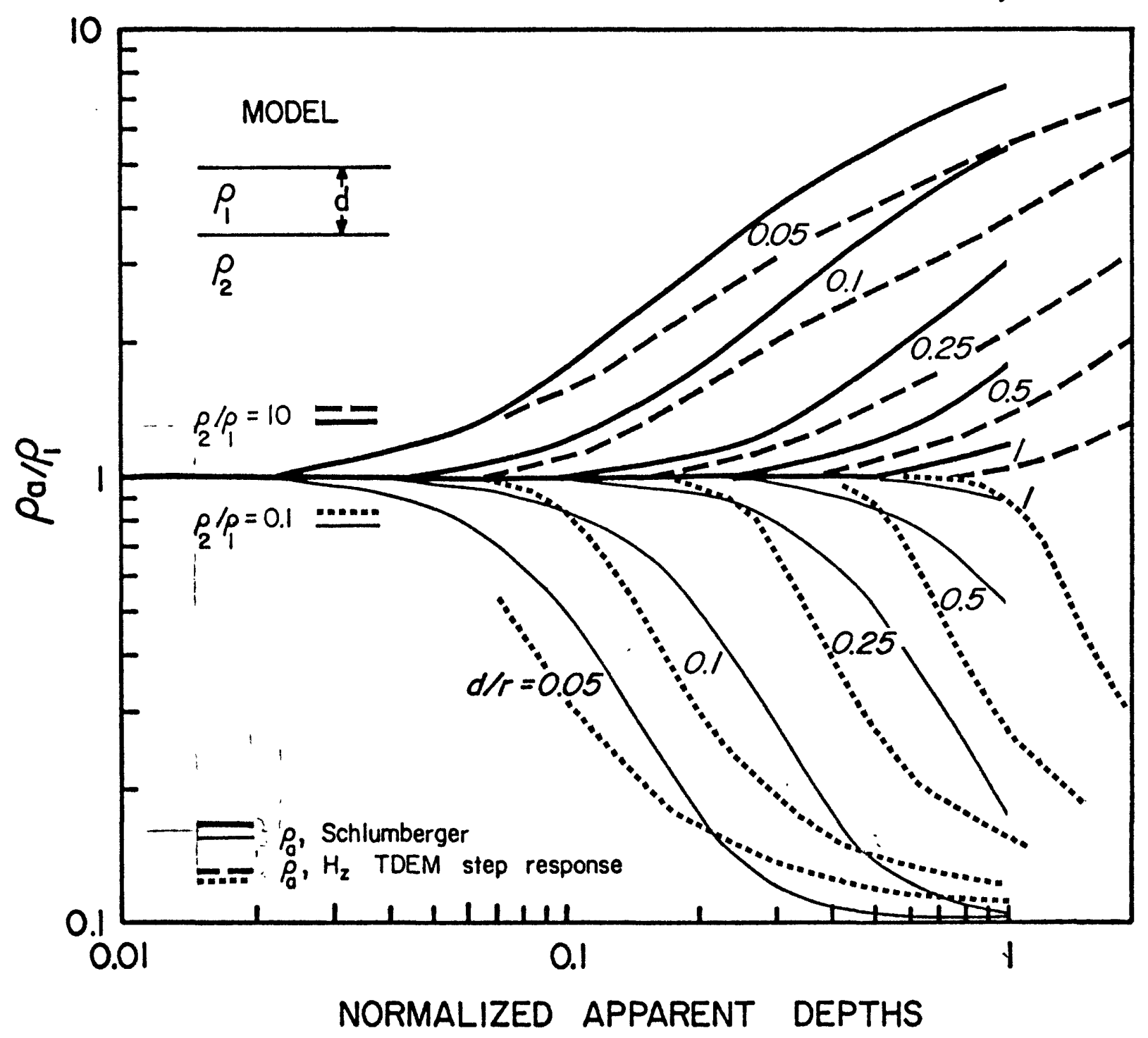

Figure 4. Solid lines are plots of normalized Schlumberger apparent resistivity versus normalized electrode spread length for conductivity contrasts of 10 and $1 / 10$ and various first layer thicknesses. Dashed lines are plots of EM apparent resistivity versus normalized apparent depth. All lengths are normalized by $r$, the source-sensor distance. 


\section{Interpretation Results \\ Using the Converted TDEM and Schlumberger Data}

The NTS TDEM sounding data have been reduced to the form described above and plotted on log-log paper in Appendix II along with the Schlumberger sounding data (locations in Figure 1) taken at the nearest location. With a few exceptions, the agreement between the TDEM and VES data, in terms of apparent resistivities and depths, is remarkable. The VES data cover electrode spacings $(A B / 2)$ of $3 \mathrm{~m}$ to $1,200 \mathrm{~m}$ whereas the TDEM apparent depths extend from about $450 \mathrm{~m}$ to over $3,000 \mathrm{~m}$. Joint inversion of both sets of data would be possible but not very efficient because of the small amount of apparent depth overlap $(400-1,200 \mathrm{~m})$. Instead, the VES data alone were inverted using program MARQDCLAG (Anderson, 1979) with the intent of matching the TDEM data with the same model. Cost of this process was around $\$ 2 /$ run as compared with $\$ 10$ to $\$ 70 /$ run for the joint inversion using MQLVTHXYZ (Kauahikaua, 1980). After just one or two runs, both sets of data were fit quite well with a single model for TD4, TD5, and VES4 (VES3 and VES5 were very similar to VES4), TD3 and VES8, TD8 and VES9, and TD6 and VES6. These models are summarized next to the VES number at the bottom of the appropriate figures in Appendix II. The parameter statistics reflect the resolution of the VES data alone.

At first, the TDEM data did not seem to be contributing any information about the earth structure that was not already indicated by the VES data. Soundings TD6 and TD7 indicate a moderate conductor at a depth greater than $3 \mathrm{~km}$, but other than the deepest few points of these two soundings, TD3, TD4, TD5, TD6, TD7, and TD8 all had the same trends as the VES data did; however, after the first few VES inversions, it was clear that the TDEM data could be reasonably fit only by a model whose basement had a resistivity significantly 
greater than that originally suggested by the VES data alone. In every case, the VES data accommodated the more resistive basement. As an example of the quantitative increase in resolution at greater depths brought about by combining the VES and TDEM sounding data, the data for TD4 and VES4 were inverted jointly. These results are presented for comparison in Appendix II. The parameter errors were decreased by significant amounts - $85 \%$ to $34 \%$ for d3 and $41 \%$ to $6 \%$ for rho3; the joint inversion cost $\$ 81$.

TDEM Computer Inversion Using Multilayer Earth Models

Soundings TD1, TD2, TD6, TD7, and TD9 were inverted with program MQLVTHXYZ. The resulting models are presented at the bottom of the appropriate figures in Appendix II, while the TDEM model responses are plotted with the original data in Appendix I. The interpreted resistivities for TD1 and TD2 are significantly lower than those for VES2. Even rigorous correction for the nearness of these two soundings to a source wire longer than $2 \mathrm{~km}$ (apparent resistivity and depth calculations implicitly assume an infinitesimally smal1 source) will raise the resistivity values by only $7 \%$. Inversions of these two data sets resolve a moderately conductive basement at 1,200-1,300 m. The data from TD6 and TD7 also suggest a conductive basement, but at significantly larger depths; in fact, the suggested depths are precisely equal to the source-sensor distance for each sounding. Inversion failed to resolve the conductive basement in the TD6 and TD7 data, therefore we may conclude that apparent resistivities from TDEM data become of questionable accuracy when apparent depths exceed the source-sensor distance. Farther to the north, TD9 resistivities are significantly higher than those in the TDEM model response computed from the VES13 model earth; however, the data and model responses are very nearly parallel, suggesting that it will be fit by an earth model with the same geometry (1ayer thicknesses) but with 
larger resistivities.

\section{Comparison of Schlumberger VES and TDEM Soundings Data and Interpretations}

In general, the two data sets compare very well. Two exceptions are the soundings nearest the source and the sounding farthest from the source (to the north). For those nearest the source, the TDEM apparent resistivities are much less than those determined from the Schlumberger data. One obvious cause for this discrepancy would be strong lateral changes in geoelectric structure. Examination of the Schlumberger data alone substantiates this hypothesis; VES1 and VES2 (the southernmost) are distinctly different from those farther north in that they do not having rising terminal branches. North of these two soundings, all VES data below 10 meters show an approximately uniform structure of 46 to $67 \mathrm{ohm}-\mathrm{m}$ overlying a basement of greater than $100 \mathrm{ohm}-\mathrm{m}$. This lateral change is reflected in the TDEM data; TD1 and TD2 (the southernmost and nearest to the source) have descending terminal branches whereas all those farther north have rising terminal branches. Unfortunately, the actual interpretations of VESI and VES2 do not compare favorably with those of TD1 and TD2. There is no way of determining which is the more accurate representation of the subsurface without other sets of electrical data. In spite of the disagreement, one could suggest that a fault exists between TD2 and VES3 on the basis of either set of data; it is uncertain whether the conductor below $1,300 \mathrm{~m}$ interpreted from the TDEM data is real or an effect of field distortion by the lateral resistivity changes.

The discrepancies noted between VES13 and TD9 at the northern edge of the study area are not as severe; both have ascending terminal branches. However, the TDEM apparent resistivities are significantly greater than 
those for the VES. Again, the discrepancy is probably due to lateral changes. The basement is generally less than $200 \mathrm{~m}$ deep in the Calico Hills themselves, dropping to several hundred meters to the north and east. The TDEM data in each of these areas underestimates the conductance of the layers above basement thereby underestimating the basement depth. TD9 is the most severe example of this, but TD3, TD7 and TD8 all show similar discrepancies when compared to nearby VES data. This must be an averaging effect due to the utilization of a fixed source to the south of the hills for the TDEM soundings.

The TDEM and VES data obtained in and near the Calico Hills agree quite closely; however, there is a distinct difference in resolution. The VES data can resolve shallow structure to 800 or $900 \mathrm{~m}$, which in this area is sufficient to resolve the conductive layers above basement (basement generally at $200 \mathrm{~m}$ ). Deeper resolution could have been achieved with electrode spacings greater than the maximum of $1,200 \mathrm{~m}$ used here. The TDEM data is not sampled at small enough times to resolve anything shallower than $800 \mathrm{~m}$ and can only provide limits on the longitudinal conductance of the layers above basement, as well as a minimum basement resistivity. The combination of the two sets of data can yield uniformly good resolution from $10 \mathrm{~m}$ to $2-3 \mathrm{~km}$. 


\section{References}

Anderson, W. L., 1979, Program MARQDCLAG: Marquardt inversion of DCSchlumberger soundings by lagged-convolution: U. S. Geological Survey Open-File Report 79-1432, 58 p.

Kauahikaua, J., 1980, Program MQLVTHXYZ: Computer inversion of threecomponent time-domain magnetic-field sounding data generated using an electric-wire source: U. S. Geological Survey Open-File Report 80-1159, $109 \mathrm{p}$.

Kauahikaua, J. and Anderson, W. L., 1977, Calculation of standard transient and frequency sounding curves for a horizontal wire source of arbitrary length: U. S. Geological Survey Open-File Report USGS-GD-77-007, 61 p., avail. from U. S. Dept. Comm. NTIS, Springfield, VA 22161 as Rept. PB-274-119.

Morrison, H, F., Phillips, K, J., and O'Brien, D. P., 1969, Quantitative interpretation of transient electromagnetic fields over a layered halfspace: Geophysical Prospecting, v. 17, p. 82-101. 
APPENDIX I: Normalized TDEM Data Plots

Solid curves are TDEM half-space model responses which are either undistorted, or distorted by a twin-T 60 Hertz notch filter (depending on how each data set was measured in the field). The resistivities corresponding to each curve are in parentheses and were obtained by multiplying each curves' unique ratio of resistivity and the square of the source-sensor distance, in $\mathrm{km}$, by the square of the actual source-sensor distance of each sounding. Data points are plotted as open circles with dots in the center, and error bars represent one standard deviation as derived by stacking. Dashed curves are the best-fit TDEM model responses; parameters and statistics for these fits are summarized in Appendix II. 


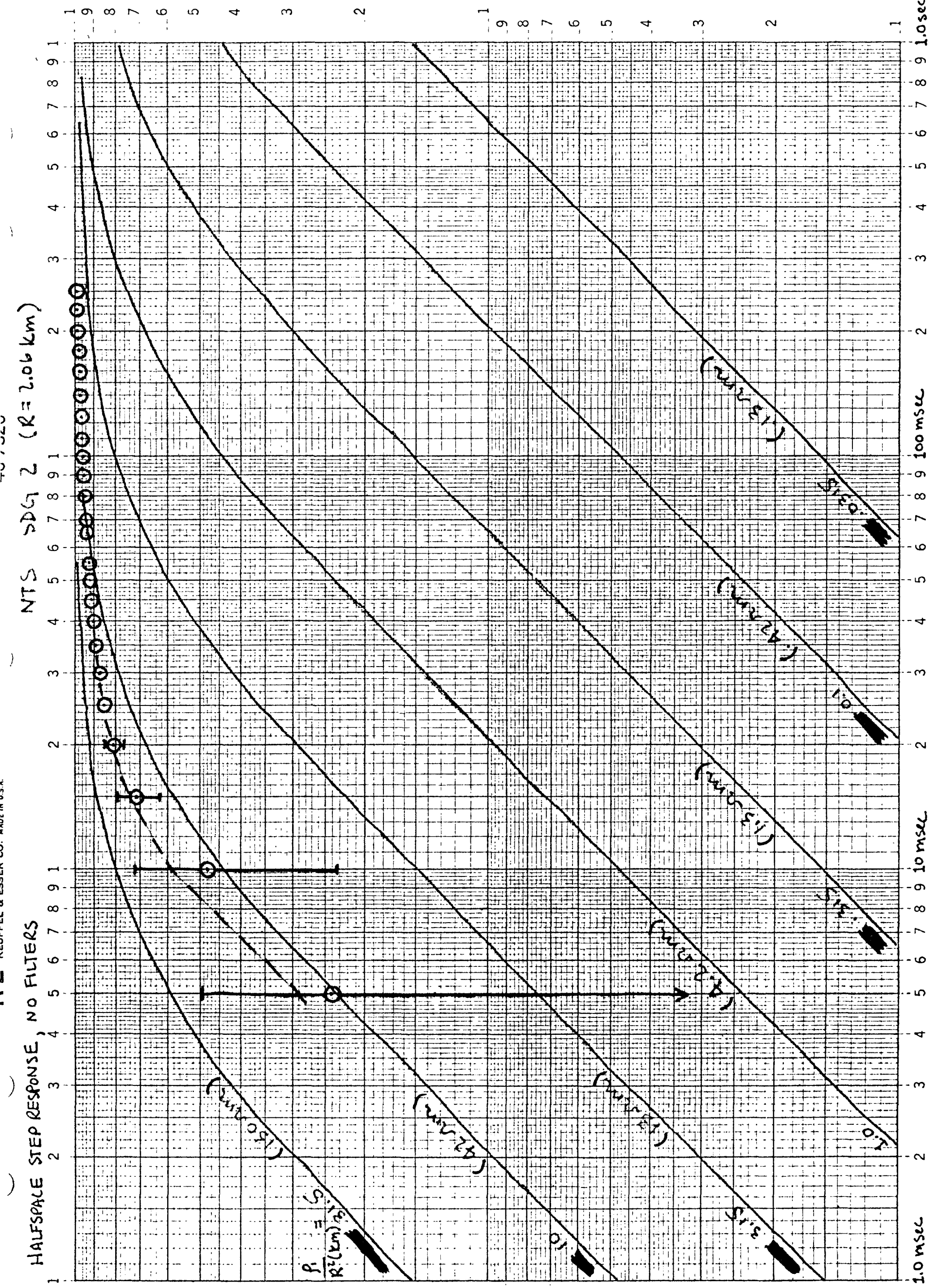




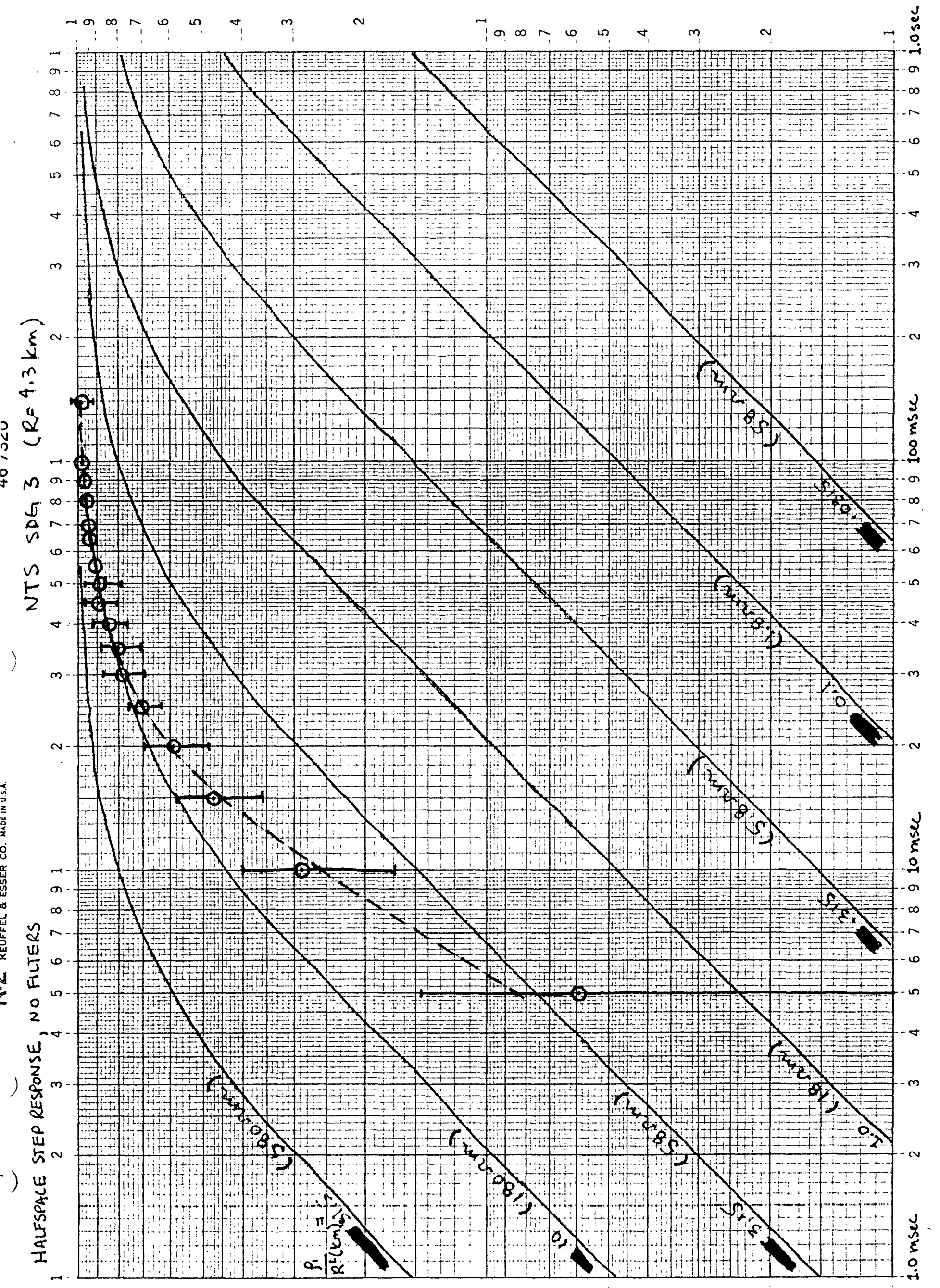




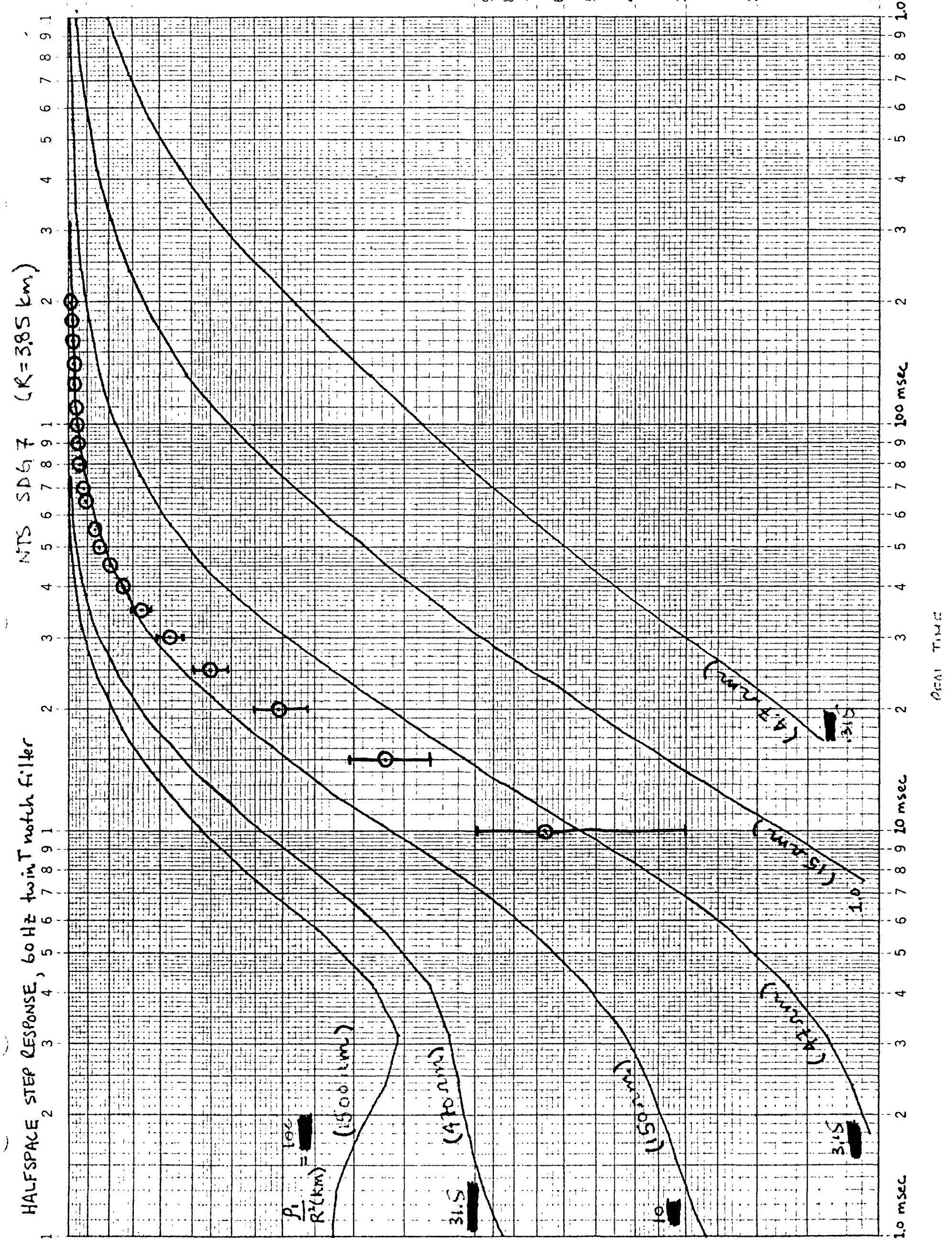




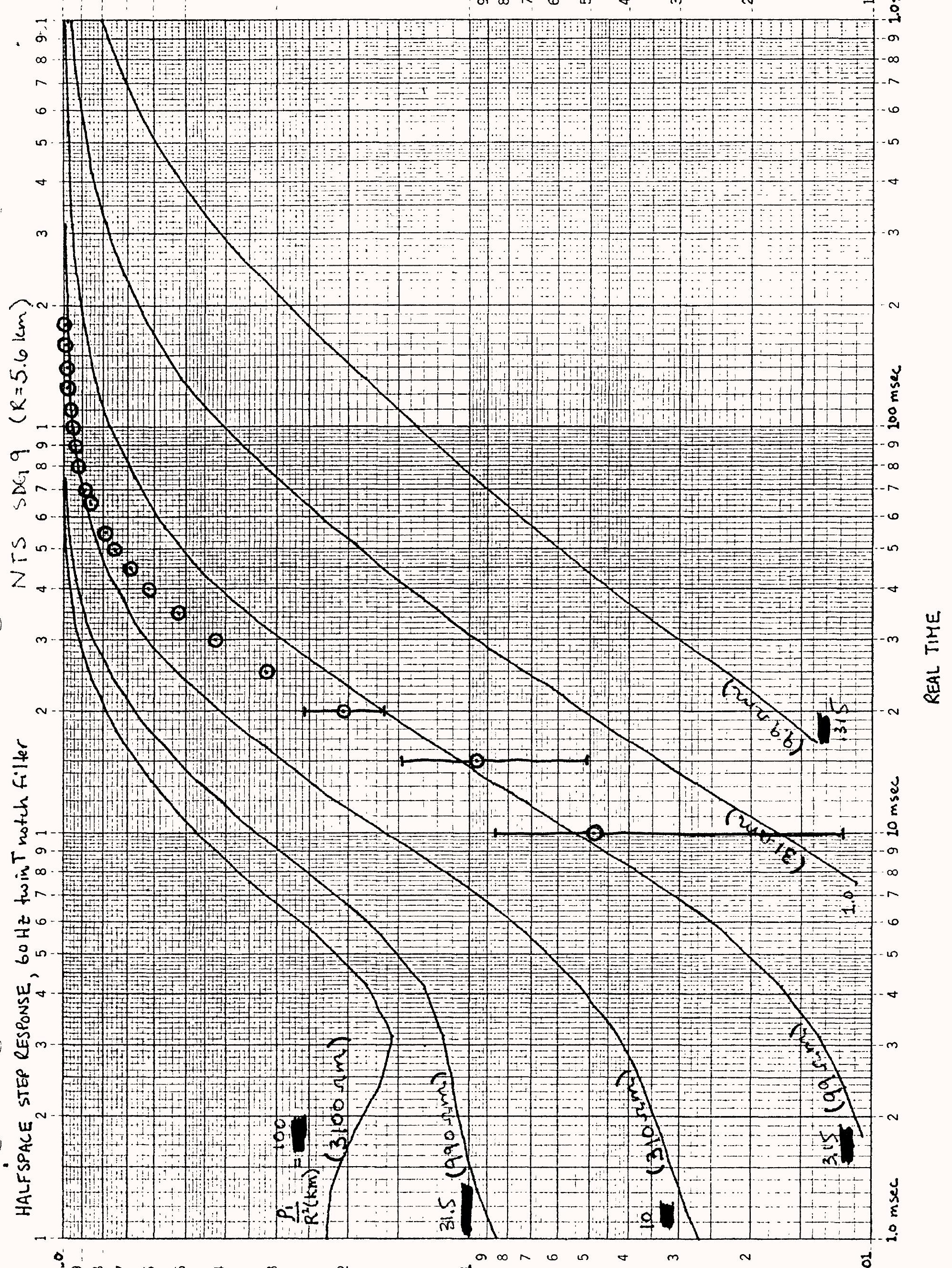


APPENDIX II: Combined Plots

of Apparent Resistivity Versus Apparent Depth

Schlumberger data are plotted as the circled symbols, and TDEM data are the uncircled symbols. Solid curves represent the best-fit Schlumberger model whose parameters are summarized in bar form at the bottom of the plot. Dashed curves represent the TDEM model response computed using the Schlumberger best-fit model parameters. The parameters listed in bar form with the TDEM station numbers are the best-fit TDEM models produced by computer inversion. The numbers accompanying the parameters are the standard parameter errors produced by the inversion program. Those that have no errors explicitly listed had errors greater than $150 \%$. An equal sign with three bars instead of two signifies a parameter that was constrained during inversion. 


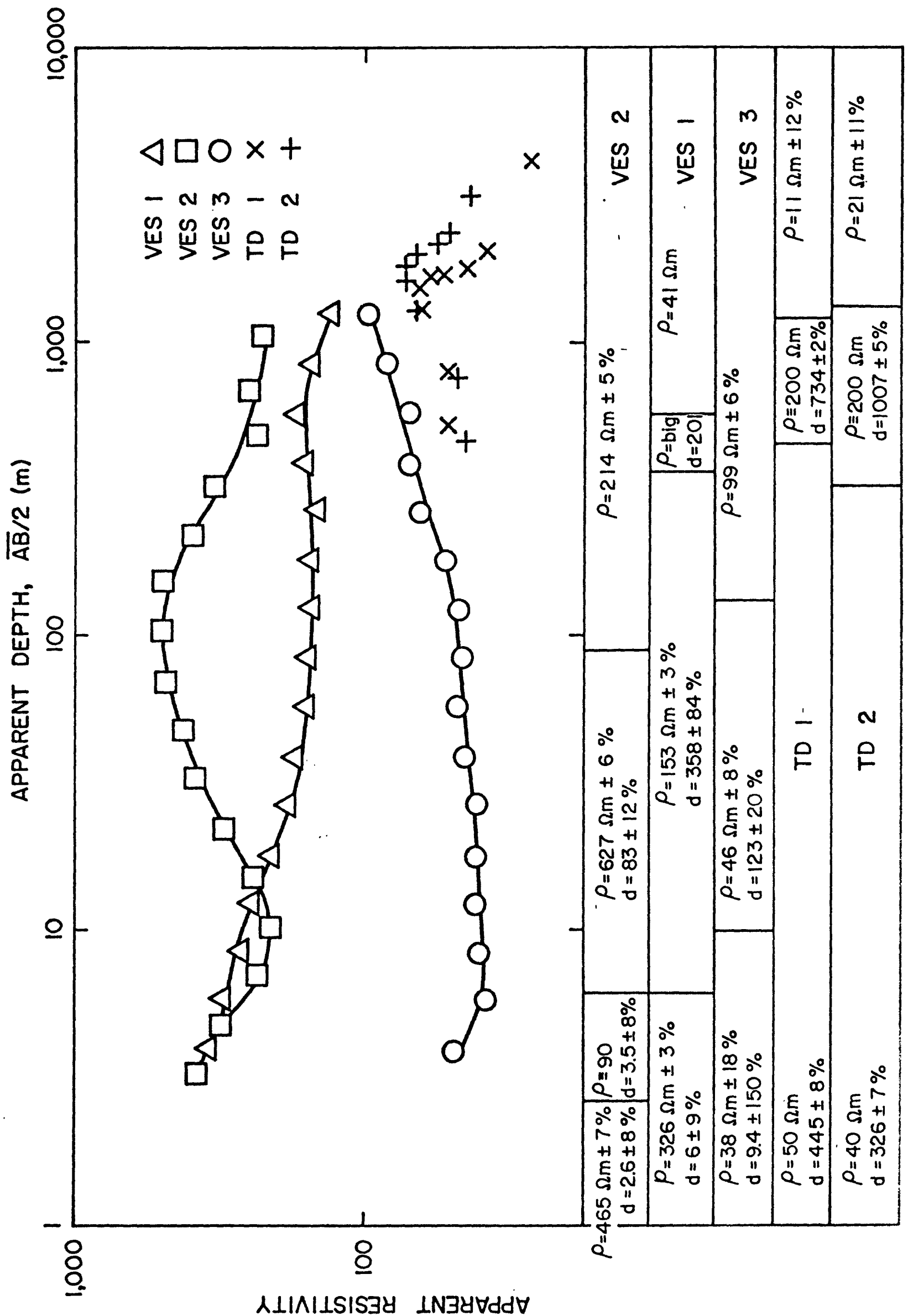




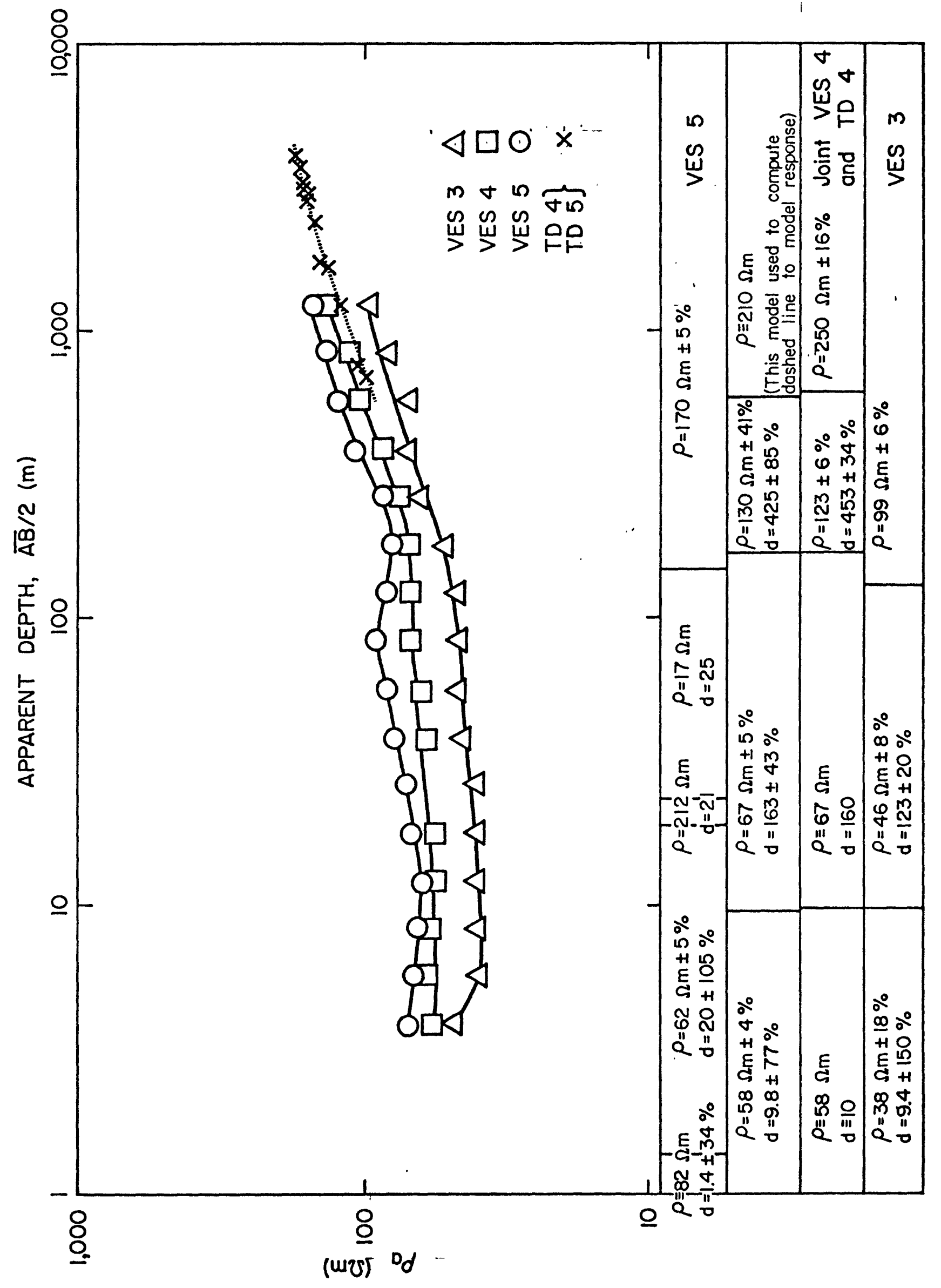




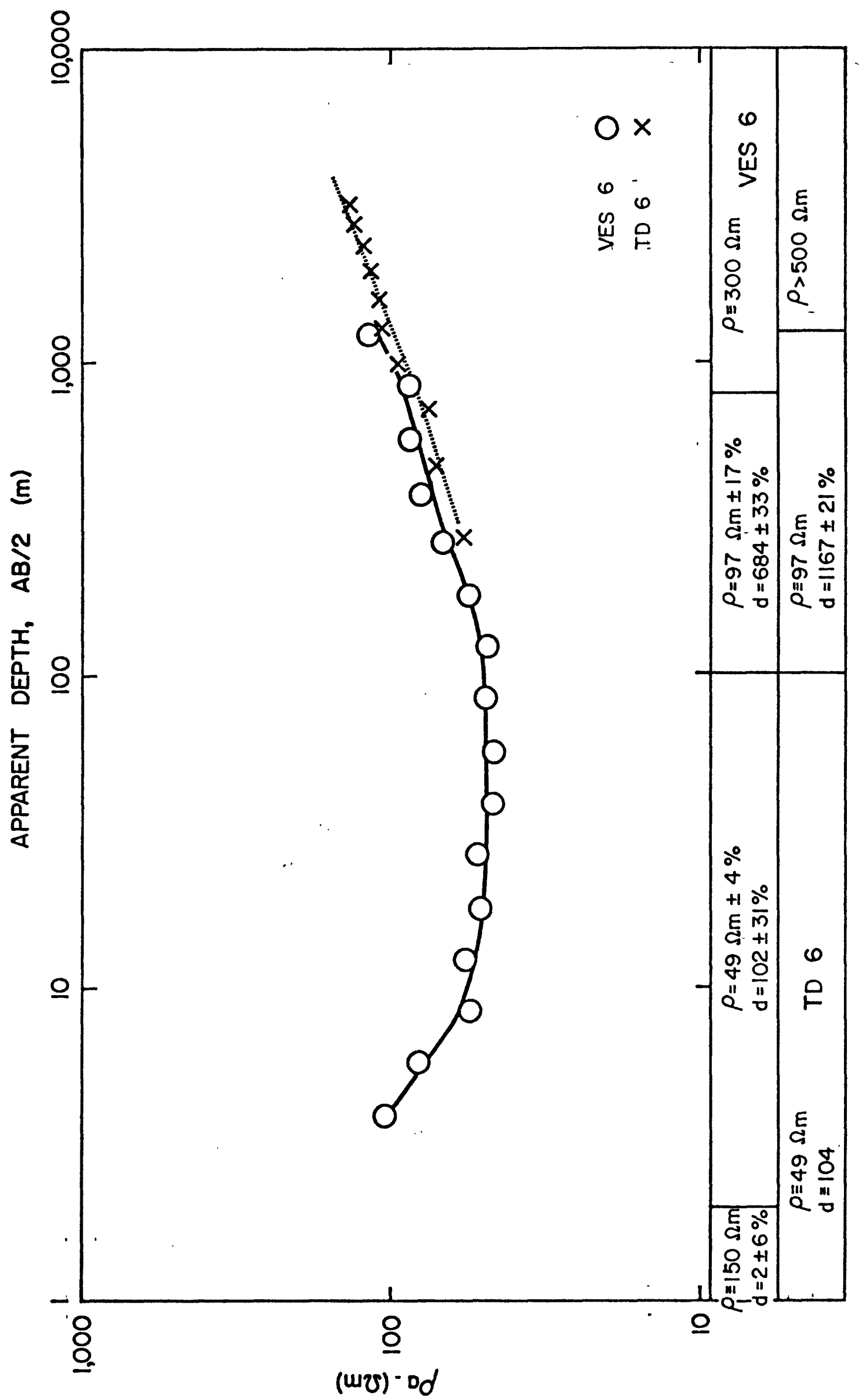




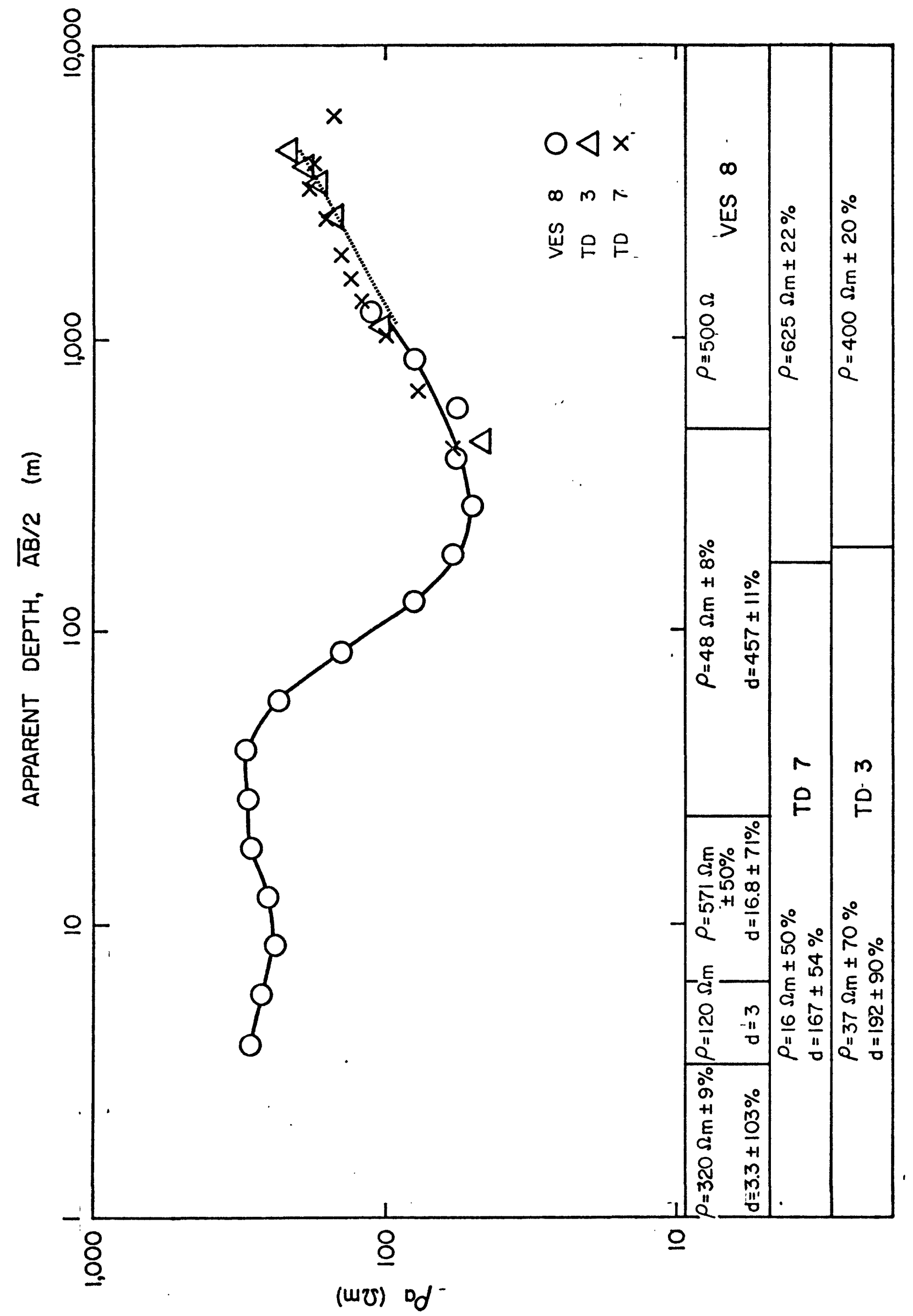




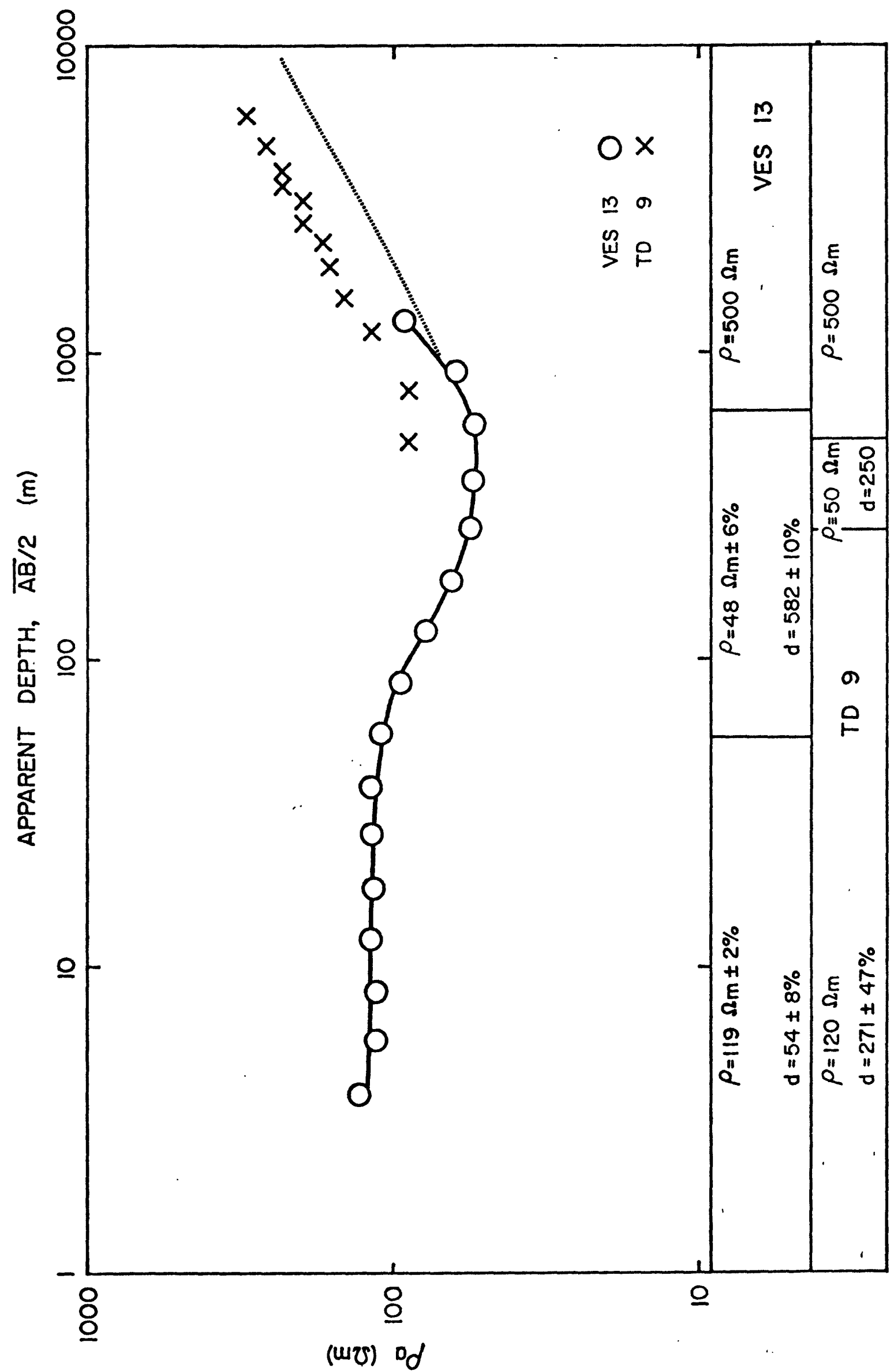

